Commun. Fac. Sci. Univ. Ank. Ser. A1 Math. Stat.

Volume 69, Number 1, Pages 668-683(2020)

DOI: $10.31801 /$ cfsuasmas. 549184

ISSN 1303-5991 E-ISSN 2618-6470

http://communications.science.ankara.edu.tr/index.php?series=A1

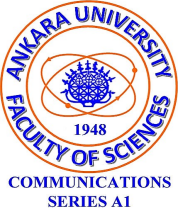

\title{
OSCILLATION RESULTS FOR SECOND ORDER HALF-LINEAR FUNCTIONAL DYNAMIC EQUATIONS WITH UNBOUNDED NEUTRAL COEFFICIENTS ON TIME SCALES
}

\author{
ORHAN ÖZDEMIR
}

\begin{abstract}
This study aims to present some new sufficient conditions for the oscillatory behavior of solutions to a class of second order half-linear functional dynamic equations with mixed neutral term i.e., the neutral term contains both retarded and advanced arguments. The results obtained are applicable in the case where the studied equation has unbounded neutral coefficients and they are new even for the linear case. Illustrative examples are also provided.
\end{abstract}

\section{INTRODUCTION}

In this study, we are concerned with the oscillation of second order half-linear mixed neutral dynamic equations of the form

$$
\left(r(t)\left(y^{\Delta}(t)\right)^{\beta}\right)^{\Delta}+\sum_{i=1}^{n} q_{i}(t) x^{\beta}\left(h_{i}(t)\right)=0, \quad t \in\left[t_{0}, \infty\right)_{\mathbb{T}}
$$

where $n \geq 1$ is an integer, $\beta$ is a ratio of positive odd integers, $\mathbb{T}$ is a time scale unbounded above with $t_{0} \in \mathbb{T}$, and

$$
y(t):=x(t)+p_{1}(t) x\left(\tau_{1}(t)\right)+p_{2}(t) x\left(\tau_{2}(t)\right) .
$$

For some basic facts on time scale calculus and dynamic equations on time scales, one may consult the excellent texts by Bohner and Peterson [8, 9]. Throughout this study it is assumed that the reader is familiar with time scale calculus, and the following conditions are always satisfied:

(i) $q_{i}:\left[t_{0}, \infty\right)_{\mathbb{T}} \rightarrow \mathbb{R}$ are nonnegative rd-continuous functions such that not all of the $q_{i}(t)$ vanish in a neighborhood of infinity for $i=1,2, \ldots, n$ and $r:\left[t_{0}, \infty\right)_{\mathbb{T}} \rightarrow \mathbb{R}$ is a positive rd-continuous function with

$$
\int_{t_{0}}^{\infty} r^{-1 / \beta}(s) \Delta s=\infty
$$

Received by the editors: April 04, 2019; Accepted: January 06, 2020.

2010 Mathematics Subject Classification. 34K11, 34K40, 34N05, 39A21.

Key words and phrases. Oscillation, second order, neutral dynamic equations, time scales. 
(ii) $\tau_{1}, \tau_{2}, h_{i}:\left[t_{0}, \infty\right)_{\mathbb{T}} \rightarrow \mathbb{T}$ are rd-continuous functions such that $\tau_{1}$ and $\tau_{2}$ are strictly increasing, $\tau_{1}(t)<t, \tau_{2}(t)>t$ and $\lim _{t \rightarrow \infty} \tau_{1}(t)=\lim _{t \rightarrow \infty} h_{i}(t)=$ $\infty$, for $i=1,2, \ldots, n$;

and, either

$\left(\mathfrak{i i i}_{\mathfrak{a}}\right) p_{1}, p_{2}:\left[t_{0}, \infty\right)_{\mathbb{T}} \rightarrow \mathbb{R}$ are rd-continuous functions with $p_{1}(t) \geq 0, p_{2}(t) \geq 1$ and $p_{2}(t) \not \equiv 1$ eventually;

or

$\left(\mathfrak{i i i}_{\mathfrak{b}}\right) p_{1}, p_{2}:\left[t_{0}, \infty\right)_{\mathbb{T}} \rightarrow \mathbb{R}$ are rd-continuous functions with $p_{2}(t) \geq 0, p_{1}(t) \geq 1$ and $p_{1}(t) \not \equiv 1$ eventually.

Wherever we write " $t \geq t_{n}$ " we mean " $t \in\left[t_{n}, \infty\right)_{\mathbb{T}}$ ".

By a solution of equation 1.1 we mean a function $x \in C_{r d}\left(\left[T_{x}, \infty\right)_{\mathbb{T}}, \mathbb{R}\right)$ which has the properties $y \in C_{r d}^{1}\left(\left[T_{x}, \infty\right)_{\mathbb{T}}, \mathbb{R}\right)$ and $r\left(y^{\Delta}\right)^{\beta} \in C_{r d}^{1}\left(\left[T_{x}, \infty\right)_{\mathbb{T}}, \mathbb{R}\right)$, and satisfies equation 1.1 on $\left[T_{x}, \infty\right)_{\mathbb{T}}$. Without further mention, we will assume throughout that every solution $x(t)$ of (1.1) under consideration here is continuable to the right and nontrivial, i.e., $x(t)$ is defined on some ray $\left[T_{x}, \infty\right)_{\mathbb{T}}$, for some $T_{x} \geq t_{0}$, and

$$
\sup \left\{|x(t)|: t \geq T_{1}\right\}>0 \quad \text { for all } T_{1} \geq T_{x} .
$$

We make the standing hypothesis that 1.1 admits such solutions. Such a solution of (1.1) is called oscillatory if it has arbitrarily large zeros on $\left[T_{x}, \infty\right)_{\mathbb{T}}$ and otherwise it is called nonoscillatory. Equation 1.1 is said to be oscillatory if all its solutions are oscillatory.

The study of oscillation of the solutions of neutral differential and difference equations presents a strong theoretical interest. One reason for this is that they arise in several areas of applied mathematics including circuit theory, bifurcation analysis, population dynamics, stability theory, the dynamics of delayed network systems and others. Besides, these equations are used in the analysis of computer networks containing lossless transmission lines, as in high speed networks where lossless transmission lines serve to connect switching circuits in the network. Also, second-order neutral delay differential equations are of great interest in biology in explaining self-balancing of the human body and in robotics in constructing biped robots [11, 13, 15, 25. Interested readers can refer to the books by Hale [15] and, Kolmanovskii and Myshkis 22] for more applications in science and technology.

Note that equation with $\mathbb{T}=\mathbb{R}, n=2, h_{1}(t)<t$ and $h_{2}(t)>t$ were encountered in the study of vibrating masses attached to an elastic bar [15, 23. Since it has some direct applications in science, the oscillatory behavior of equation (1.1) and it's special and more general forms have been studied by numerous authors utilizing different methods. In reviewing the related literature, most of such results are concerned with the cases where the functions $p_{j}(t)$ are constant or bounded functions, for $j=1,2$; see for example [6, 10, 12, 16, 19, 23, 28, 30, 36, 37] and the 
references cited therein. However, to the best of our knowledge, there does not appear to be any oscillation results for second order mixed neutral dynamic equations in the case where the neutral term includes unbounded neutral coefficients.

Motivated by the papers mentioned above, the purpose of this study is to establish some new oscillation criteria that can be applied in the case where equation (1.1) has unbounded neutral coefficients, that is, $p_{1}(t) \rightarrow \infty$ and/or $p_{2}(t) \rightarrow \infty$ as $t \rightarrow \infty$. Moreover, it should be also pointed out that the results obtained here are new even for $\mathbb{T}=\mathbb{R}$ and $\mathbb{T}=\mathbb{Z}$, for the linear case when $\beta=1$, for $r(t)=1$, for $n=1$ and for discrete deviating arguments such as $\tau_{1}(t)=t-a, \tau_{2}(t)=t+b$ and $h_{i}(t)=t \mp c$ with $a, b, c>0$ and $i=1,2, \ldots, n$.

For convenience, we will use the following notations:

$$
\gamma=\frac{\beta+1}{\beta}, \quad \theta_{+}(t):=\max \{0, \theta(t)\} \quad \text { and } A\left(t, t_{1}\right):=\int_{t_{1}}^{t} r^{-1 / \beta}(s) \Delta s
$$

for any rd-continuous function $\theta$.

The following lemma is required in our main results. Since the proof is standard we omit the details here.

Lemma 1.1. Assume that conditions $(\mathfrak{i})-\left(\mathfrak{i i i}_{\mathfrak{a}}\right)\left(\right.$ or $(\mathfrak{i}),(\mathfrak{i i})$, ( $\left.\left.\mathfrak{i i}_{\mathfrak{b}}\right)\right)$ hold and $x(t)$ is an eventually positive solution of (1.1). Then $y(t)$ satisfies $y(t)>0, y^{\Delta}(t)>0$ and $\left(r(t)\left(y^{\Delta}(t)\right)^{\beta}\right)^{\Delta}<0$, for all $t$ large enough.

Lemma 1.2. 17] If $X$ and $Y$ are nonnegative and $\lambda>1$, then

$$
\lambda X Y^{\lambda-1}-X^{\lambda} \leq(\lambda-1) Y^{\lambda},
$$

where equality holds if and only if $X=Y$.

\section{Oscillation RESUlts When $\left(\mathfrak{i i i}_{\mathfrak{a}}\right)$ HOLDS}

In this section, we establish some new criteria for the oscillation of $\sqrt{1.1}$ in the cases where $h_{i}(t) \leq \tau_{2}(t)$ and $h_{i}(t) \geq \tau_{2}(t)$ for $i=1,2, \ldots, n$, respectively. For notational purposes, we let

$$
\psi(t):=\frac{1}{p_{2}\left(\tau_{2}^{-1}(t)\right)}\left[1-\frac{1}{p_{2}\left(\tau_{2}^{-1}\left(\tau_{2}^{-1}(t)\right)\right)}-\frac{p_{1}\left(\tau_{2}^{-1}(t)\right)}{p_{2}\left(\tau_{2}^{-1}\left(\tau_{1}\left(\tau_{2}^{-1}(t)\right)\right)\right)}\right]
$$

where $\tau_{2}^{-1}$ denotes the inverse function of $\tau_{2}$, and throughout this section we assume that $\psi(t)>0$ for all sufficiently large $t$.

Theorem 2.1. Assume that conditions $(\mathfrak{i})$-(iii $\left.\mathfrak{i a}_{\mathfrak{a}}\right)$ hold and $h_{i}(t) \leq \tau_{2}(t)$ for $i=$ $1,2, \ldots, n$. If there exists a positive function $\eta \in C_{r d}^{1}\left(\left[t_{0}, \infty\right)_{\mathbb{T}}, \mathbb{R}\right)$ such that

$$
\limsup _{t \rightarrow \infty} \int_{T}^{t}\left(\Psi_{1}(s)-\frac{r(s)\left(\eta^{\Delta}(s)\right)^{\beta+1}}{(\beta+1)^{\beta+1} \eta^{\beta}(s)}\right) \Delta s=\infty
$$


for $T>t_{1}$ where $t_{1} \in\left[t_{0}, \infty\right)_{\mathbb{T}}$ sufficiently large and

$$
\Psi_{1}(t)=\eta(t)\left(\sum_{i=1}^{n} q_{i}(t) \psi^{\beta}\left(h_{i}(t)\right) \frac{A^{\beta}\left(\tau_{2}^{-1}\left(h_{i}(t)\right), t_{1}\right)}{A^{\beta}\left(t, t_{1}\right)}\right),
$$

then equation (1.1) is oscillatory.

Proof. Let $x(t)$ be a nonoscillatory solution of (1.1). Without loss of generality, we may assume that there exists $t_{1} \in\left[t_{0}, \infty\right)_{\mathbb{T}}$ such that $x(t)>0, x\left(\tau_{1}(t)\right)>0$, $x\left(\tau_{2}(t)\right)>0$ and $x\left(h_{i}(t)\right)>0$ for $t \geq t_{1}$ and $i=1,2, \ldots, n$. The proof if $x(t)$ is eventually negative is similar, so we omit the details of that case here as well as in the remaining proofs in this paper. In view of Lemma 1.1, it is obvious that

$$
y(t)>0, y^{\Delta}(t)>0 \text { and }\left(r(t)\left(y^{\Delta}(t)\right)^{\beta}\right)^{\Delta}<0 \text { for } t \geq t_{1} .
$$

Since $r(t)\left(y^{\Delta}(t)\right)^{\beta}$ is decreasing, we have

$$
\begin{aligned}
y(t) & =y\left(t_{1}\right)+\int_{t_{1}}^{t} \frac{\left(r(s)\left(y^{\Delta}(s)\right)^{\beta}\right)^{1 / \beta}}{r^{1 / \beta}(s)} \Delta s \\
& \geq\left(r(t)\left(y^{\Delta}(t)\right)^{\beta}\right)^{1 / \beta} A\left(t, t_{1}\right) \quad \text { for } t \geq t_{1} .
\end{aligned}
$$

Thus

$$
\left(\frac{y(t)}{A\left(t, t_{1}\right)}\right)^{\Delta} \leq 0
$$

i.e., $y(t) / A\left(t, t_{1}\right)$ is nonincreasing on $\left[t_{1}, \infty\right)_{\mathbb{T}}$. From the definition of $y(t)$, we get

$$
\begin{aligned}
x(t)= & \frac{1}{p_{2}\left(\tau_{2}^{-1}(t)\right)}\left(y\left(\tau_{2}^{-1}(t)\right)-x\left(\tau_{2}^{-1}(t)\right)-p_{1}\left(\tau_{2}^{-1}(t)\right) x\left(\tau_{1}\left(\tau_{2}^{-1}(t)\right)\right)\right) \\
= & \frac{y\left(\tau_{2}^{-1}(t)\right)}{p_{2}\left(\tau_{2}^{-1}(t)\right)}-\frac{y\left(\tau_{2}^{-1}\left(\tau_{2}^{-1}(t)\right)\right)-x\left(\tau_{2}^{-1}\left(\tau_{2}^{-1}(t)\right)\right)}{p_{2}\left(\tau_{2}^{-1}(t)\right) p_{2}\left(\tau_{2}^{-1}\left(\tau_{2}^{-1}(t)\right)\right)} \\
& +\frac{p_{1}\left(\tau_{2}^{-1}\left(\tau_{2}^{-1}(t)\right)\right) x\left(\tau_{1}\left(\tau_{2}^{-1}\left(\tau_{2}^{-1}(t)\right)\right)\right)}{p_{2}\left(\tau_{2}^{-1}(t)\right) p_{2}\left(\tau_{2}^{-1}\left(\tau_{2}^{-1}(t)\right)\right)} \\
& -\frac{p_{1}\left(\tau_{2}^{-1}(t)\right)}{p_{2}\left(\tau_{2}^{-1}(t)\right)}\left[\frac{y\left(\tau_{2}^{-1}\left(\tau_{1}\left(\tau_{2}^{-1}(t)\right)\right)\right)-x\left(\tau_{2}^{-1}\left(\tau_{1}\left(\tau_{2}^{-1}(t)\right)\right)\right)}{p_{2}\left(\tau_{2}^{-1}\left(\tau_{1}\left(\tau_{2}^{-1}(t)\right)\right)\right)}\right] \\
& \left.-\frac{p_{1}\left(\tau_{2}^{-1}\left(\tau_{1}\left(\tau_{2}^{-1}(t)\right)\right) x\left(\tau_{1}\left(\tau_{2}^{-1}\left(\tau_{1}\left(\tau_{2}^{-1}(t)\right)\right)\right)\right)\right.}{p_{2}\left(\tau_{2}^{-1}\left(\tau_{1}\left(\tau_{2}^{-1}(t)\right)\right)\right)}\right] \\
\geq & \frac{y\left(\tau_{2}^{-1}(t)\right)}{p_{2}\left(\tau_{2}^{-1}(t)\right)}-\frac{y\left(\tau_{2}^{-1}\left(\tau_{2}^{-1}(t)\right)\right)}{p_{2}\left(\tau_{2}^{-1}(t)\right) p_{2}\left(\tau_{2}^{-1}\left(\tau_{2}^{-1}(t)\right)\right)}-\frac{p_{1}\left(\tau_{2}^{-1}(t)\right) y\left(\tau_{2}^{-1}\left(\tau_{1}\left(\tau_{2}^{-1}(t)\right)\right)\right)}{p_{2}\left(\tau_{2}^{-1}(t)\right) p_{2}\left(\tau_{2}^{-1}\left(\tau_{1}\left(\tau_{2}^{-1}(t)\right)\right)\right)} .
\end{aligned}
$$

Using the fact that the functions $y, \tau_{1}$ and $\tau_{2}$ are strictly increasing, and noting that $\tau_{1}(t)<t<\tau_{2}(t)$, we get

$$
y\left(\tau_{2}^{-1}\left(\tau_{2}^{-1}(t)\right)\right)<y\left(\tau_{2}^{-1}(t)\right)
$$


and

$$
y\left(\tau_{2}^{-1}\left(\tau_{1}\left(\tau_{2}^{-1}(t)\right)\right)\right)<y\left(\tau_{2}^{-1}(t)\right) .
$$

Using (2.5) and (2.6) in (2.4) gives

$$
x(t) \geq \frac{1}{p_{2}\left(\tau_{2}^{-1}(t)\right)}\left[1-\frac{1}{p_{2}\left(\tau_{2}^{-1}\left(\tau_{2}^{-1}(t)\right)\right)}-\frac{p_{1}\left(\tau_{2}^{-1}(t)\right)}{p_{2}\left(\tau_{2}^{-1}\left(\tau_{1}\left(\tau_{2}^{-1}(t)\right)\right)\right)}\right] y\left(\tau_{2}^{-1}(t)\right)
$$

for $t \geq t_{1}$. Since $\lim _{t \rightarrow \infty} h_{i}(t)=\infty$, we can choose $t_{2} \geq t_{1}$ such that all $h_{i}(t) \geq t_{1}$ for $t \geq t_{2}$, where $i=1,2, \ldots, n$. Thus, from 2.7) we obtain

$$
x\left(h_{i}(t)\right) \geq \psi\left(h_{i}(t)\right) y\left(\tau_{2}^{-1}\left(h_{i}(t)\right)\right), \quad i=1,2, \ldots, n
$$

for $t \geq t_{2}$. Using (2.8) in (1.1) gives

$$
\left(r(t)\left(y^{\Delta}(t)\right)^{\beta}\right)^{\Delta}+\sum_{i=1}^{n} q_{i}(t) \psi^{\beta}\left(h_{i}(t)\right) y^{\beta}\left(\tau_{2}^{-1}\left(h_{i}(t)\right)\right) \leq 0 .
$$

Define the function $\omega(t)$ by the Riccati substitution

$$
\omega(t):=\eta(t) \frac{r(t)\left(y^{\Delta}(t)\right)^{\beta}}{y^{\beta}(t)} \quad \text { for } t \geq t_{2} .
$$

Clearly $\omega(t)>0$ and from 2.9 we see that

$$
\begin{aligned}
\omega^{\Delta}(t)= & \frac{\eta(t)}{y^{\beta}(t)}\left(r(t)\left(y^{\Delta}(t)\right)^{\beta}\right)^{\Delta}+\left(\frac{\eta(t)}{y^{\beta}(t)}\right)^{\Delta} r(\sigma(t))\left(y^{\Delta}(\sigma(t))\right)^{\beta} \\
\leq & \eta^{\Delta}(t) \frac{r(\sigma(t))\left(y^{\Delta}(\sigma(t))\right)^{\beta}}{y^{\beta}(\sigma(t))}-\eta(t) \frac{r(\sigma(t))\left(y^{\Delta}(\sigma(t))\right)^{\beta}\left(y^{\beta}(t)\right)^{\Delta}}{y^{\beta}(t) y^{\beta}(\sigma(t))} \\
& -\eta(t)\left(\sum_{i=1}^{n} q_{i}(t) \psi^{\beta}\left(h_{i}(t)\right) \frac{y^{\beta}\left(\tau_{2}^{-1}\left(h_{i}(t)\right)\right)}{y^{\beta}(t)}\right) \quad \text { for } t \geq t_{2},
\end{aligned}
$$

where $\sigma(t)$ is the forward jump operator on time scale $\mathbb{T}$. Using the fact $y(t) / A\left(t, t_{1}\right)$ is nonincreasing, and noting that $h_{i}(t) \leq \tau_{2}(t)$ implies $\tau_{2}^{-1}\left(h_{i}(t)\right) \leq t$, we obtain

$$
\frac{y\left(\tau_{2}^{-1}\left(h_{i}(t)\right)\right)}{y(t)} \geq \frac{A\left(\tau_{2}^{-1}\left(h_{i}(t)\right), t_{1}\right)}{A\left(t, t_{1}\right)} .
$$

for $i=1,2, \ldots, n$. Substituting 2.12 into 2.11 gives

$$
\begin{aligned}
\omega^{\Delta}(t) \leq & \eta^{\Delta}(t) \frac{r(\sigma(t))\left(y^{\Delta}(\sigma(t))\right)^{\beta}}{y^{\beta}(\sigma(t))}-\eta(t) \frac{r(\sigma(t))\left(y^{\Delta}(\sigma(t))\right)^{\beta}\left(y^{\beta}(t)\right)^{\Delta}}{y^{\beta}(t) y^{\beta}(\sigma(t))} \\
& -\eta(t)\left(\sum_{i=1}^{n} q_{i}(t) \psi^{\beta}\left(h_{i}(t)\right) \frac{A^{\beta}\left(\tau_{2}^{-1}\left(h_{i}(t)\right), t_{1}\right)}{A^{\beta}\left(t, t_{1}\right)}\right) \quad \text { for } t \geq t_{2} .
\end{aligned}
$$


From [8, Theorem 1.90], we obtain

$$
\left(y^{\beta}(t)\right)^{\Delta} \geq\left\{\begin{array}{l}
\beta y^{\beta-1}(\sigma(t)) y^{\Delta}(t), \quad \text { if } 0<\beta \leq 1 \\
\beta y^{\beta-1}(t) y^{\Delta}(t), \quad \text { if } \beta>1
\end{array}\right.
$$

If $0<\beta \leq 1$, then we have from 2.13) and 2.14 that

$$
\begin{aligned}
\omega^{\Delta}(t) \leq & \eta^{\Delta}(t) \frac{r(\sigma(t))\left(y^{\Delta}(\sigma(t))\right)^{\beta}}{y^{\beta}(\sigma(t))}-\beta \eta(t) \frac{r(\sigma(t))\left(y^{\Delta}(\sigma(t))\right)^{\beta} y^{\Delta}(t)}{y^{\beta}(t) y(\sigma(t))} \\
& -\eta(t)\left(\sum_{i=1}^{n} q_{i}(t) \psi^{\beta}\left(h_{i}(t)\right) \frac{A^{\beta}\left(\tau_{2}^{-1}\left(h_{i}(t)\right), t_{1}\right)}{A^{\beta}\left(t, t_{1}\right)}\right)
\end{aligned}
$$

If $\beta>1$, then we have from 2.13 and 2.14 that

$$
\begin{aligned}
\omega^{\Delta}(t) \leq & \eta^{\Delta}(t) \frac{r(\sigma(t))\left(y^{\Delta}(\sigma(t))\right)^{\beta}}{y^{\beta}(\sigma(t))}-\beta \eta(t) \frac{r(\sigma(t))\left(y^{\Delta}(\sigma(t))\right)^{\beta} y^{\Delta}(t)}{y(t) y^{\beta}(\sigma(t))} \\
& -\eta(t)\left(\sum_{i=1}^{n} q_{i}(t) \psi^{\beta}\left(h_{i}(t)\right) \frac{A^{\beta}\left(\tau_{2}^{-1}\left(h_{i}(t)\right), t_{1}\right)}{A^{\beta}\left(t, t_{1}\right)}\right) .
\end{aligned}
$$

Using the fact that $y(t)$ is increasing and $r(t)\left(y^{\Delta}(t)\right)^{\beta}$ is decreasing, we get $y(t) \leq$ $y(\sigma(t))$ and $y^{\Delta}(t) \geq r^{1 / \beta}(\sigma(t)) y^{\Delta}(\sigma(t)) / r^{1 / \beta}(t)$, respectively. Thus, combining 2.15) and 2.16 we obtain for $\beta>0$ and $t \geq t_{2}$,

$$
\begin{aligned}
\omega^{\Delta}(t) \leq & \eta^{\Delta}(t) \frac{r(\sigma(t))\left(y^{\Delta}(\sigma(t))\right)^{\beta}}{y^{\beta}(\sigma(t))}-\beta \eta(t) \frac{r^{\gamma}(\sigma(t))\left(y^{\Delta}(\sigma(t))\right)^{\beta+1}}{r^{1 / \beta}(t) y^{\beta+1}(\sigma(t))} \\
& -\eta(t)\left(\sum_{i=1}^{n} q_{i}(t) \psi^{\beta}\left(h_{i}(t)\right) \frac{A^{\beta}\left(\tau_{2}^{-1}\left(h_{i}(t)\right), t_{1}\right)}{A^{\beta}\left(t, t_{1}\right)}\right) \\
= & \eta^{\Delta}(t) \frac{\omega(\sigma(t))}{\eta(\sigma(t))}-\beta \frac{\eta(t)}{r^{1 / \beta}(t)} \frac{\omega^{\gamma}(\sigma(t))}{\eta^{\gamma}(\sigma(t))} \\
& -\eta(t)\left(\sum_{i=1}^{n} q_{i}(t) \psi^{\beta}\left(h_{i}(t)\right) \frac{A^{\beta}\left(\tau_{2}^{-1}\left(h_{i}(t)\right), t_{1}\right)}{A^{\beta}\left(t, t_{1}\right)}\right) .
\end{aligned}
$$

If we apply Lemma 1.2 with

$$
X=\frac{[\beta \eta(t)]^{1 / \gamma}}{\left[r^{1 / \beta}(t) \eta^{\gamma}(\sigma(t))\right]^{1 / \gamma}} \omega(\sigma(t)) \text { and } Y=\left[\frac{\beta}{\beta+1} \frac{\left[r^{1 / \beta}(t) \eta^{\gamma}(\sigma(t))\right]^{1 / \gamma}}{[\beta \eta(t)]^{1 / \gamma}} \frac{\eta^{\Delta}(t)}{\eta(\sigma(t))}\right]^{\beta}
$$

we see that

$$
\eta^{\Delta}(t) \frac{\omega(\sigma(t))}{\eta(\sigma(t))}-\beta \frac{\eta(t)}{r^{1 / \beta}(t)} \frac{\omega^{\gamma}(\sigma(t))}{\eta^{\gamma}(\sigma(t))} \leq \frac{1}{(\beta+1)^{\beta+1}} \frac{r(t)\left(\eta^{\Delta}(t)\right)^{\beta+1}}{\eta^{\beta}(t)} .
$$


Using 2.18 in 2.17) gives

$$
\omega^{\Delta}(t) \leq \frac{r(t)\left(\eta^{\Delta}(t)\right)^{\beta+1}}{(\beta+1)^{\beta+1} \eta^{\beta}(t)}-\eta(t)\left(\sum_{i=1}^{n} q_{i}(t) \psi^{\beta}\left(h_{i}(t)\right) \frac{A^{\beta}\left(\tau_{2}^{-1}\left(h_{i}(t)\right), t_{1}\right)}{A^{\beta}\left(t, t_{1}\right)}\right) .
$$

Integrating the latter inequality from $t_{2}$ to $t$ yields

$$
\int_{t_{2}}^{t}\left(\eta(s)\left(\sum_{i=1}^{n} q_{i}(s) \psi^{\beta}\left(h_{i}(s)\right) \frac{A^{\beta}\left(\tau_{2}^{-1}\left(h_{i}(s)\right), t_{1}\right)}{A^{\beta}\left(s, t_{1}\right)}\right)-\frac{r(s)\left(\eta^{\Delta}(s)\right)^{\beta+1}}{(\beta+1)^{\beta+1} \eta^{\beta}(s)}\right) \Delta s \leq \omega\left(t_{2}\right)
$$

which contradicts 2.1] and completes the proof.

Following, we give an oscillation criterion for (1.1) by using the integral averaging technique due to Philos [27. First we need to introduce, the function class $\mathcal{P}$. Let $D_{0} \equiv\left\{(t, s) \in \mathbb{T}^{2}: t>s \geq t_{0}\right\}, D \equiv\left\{(t, s) \in \mathbb{T}^{2}: t \geq s \geq t_{0}\right\}$ and $H, h \in C_{r d}(D, \mathbb{R})$. The function $H \in C_{r d}(D, \mathbb{R})$ is said to belongs to the class $\mathcal{P}$ if

$\left(\mathcal{P}_{1}\right) H(t, t)=0$ for $t \geq t_{0}$ and $H(t, s)>0$ on $D_{0}$,

$\left(\mathcal{P}_{2}\right) H$ has a nonpositive rd-continuous $\Delta$-partial derivative $H^{\Delta_{s}}(t, s)$ on $D_{0}$ with respect to second variable and satisfies

$$
H^{\Delta_{s}}(t, s)+H(t, s) \frac{\eta^{\Delta}(s)}{\eta(\sigma(s))}=\frac{h(t, s)}{\eta(\sigma(s))} H^{1 / \gamma}(t, s),
$$

where the function $\eta$ is as in Theorem 2.1

Theorem 2.2. Assume that conditions $(\mathfrak{i})-\left(\mathfrak{i i i}_{\mathfrak{a}}\right)$ hold and $h_{i}(t) \leq \tau_{2}(t)$ for $i=$ $1,2, \ldots, n$. Suppose also that there exist functions $\eta \in C_{r d}^{1}\left(\left[t_{0}, \infty\right)_{\mathbb{T}}, \mathbb{R}^{+}\right)$and $H, h \in$ $C_{r d}(D, \mathbb{R})$ with $H$ belongs to the class $\mathcal{P}$ such that

$$
\limsup _{t \rightarrow \infty} \frac{1}{H\left(t, t_{*}\right)} \int_{t_{*}}^{t}\left[H(t, s) \Psi_{1}(s)-\frac{r(s)\left(h_{+}(t, s)\right)^{\beta+1}}{(\beta+1)^{\beta+1} \eta^{\beta}(s)}\right] \Delta s=\infty,
$$

where $\Psi_{1}(t)$ is as in Theorem 2.1 and $t_{*}>t_{1}$ for sufficiently large $t_{1} \in\left[t_{0}, \infty\right)_{\mathbb{T}}$. Then every solution of equation (1.1) is oscillatory.

Proof. Let $x(t)$ be a nonoscillatory solution of 1.1). Without loss of generality, we may assume that there exists $t_{1} \in\left[t_{0}, \infty\right)_{\mathbb{T}}$ such that $x(t)>0, x\left(\tau_{1}(t)\right)>0$, $x\left(\tau_{2}(t)\right)>0$ and $x\left(h_{i}(t)\right)>0$ for $t \geq t_{1}$ and $i=1,2, \ldots, n$. Proceeding as in the proof of Theorem 2.1. we again arrive at 2.17) for $t \geq t_{2}$. In view of $\left(\mathcal{P}_{1}\right)$ and $\left(\mathcal{P}_{2}\right)$, it follows from 2.17) that

$$
\begin{array}{r}
\int_{t_{2}}^{t} H(t, s) \eta(s)\left(\sum_{i=1}^{n} q_{i}(s) \psi^{\beta}\left(h_{i}(s)\right) \frac{A^{\beta}\left(\tau_{2}^{-1}\left(h_{i}(s)\right), t_{1}\right)}{A^{\beta}\left(s, t_{1}\right)}\right) \Delta s \leq-\int_{t_{2}}^{t} H(t, s) \omega^{\Delta}(s) \Delta s \\
+\int_{t_{2}}^{t} H(t, s) \eta^{\Delta}(s) \frac{\omega(\sigma(s))}{\eta(\sigma(s))} \Delta s-\int_{t_{2}}^{t} H(t, s) \beta \frac{\eta(s)}{r^{1 / \beta}(s)} \frac{\omega^{\gamma}(\sigma(s))}{\eta^{\gamma}(\sigma(s))} \Delta s .
\end{array}
$$


Using the integration by parts formula on time scales, we obtain

$$
\begin{array}{r}
\int_{t_{2}}^{t} H(t, s) \Psi_{1}(s) \Delta s \leq H\left(t, t_{2}\right) \omega\left(t_{2}\right)+\int_{t_{2}}^{t} H^{\Delta_{s}}(t, s) \omega(\sigma(s)) \Delta s \\
+\int_{t_{2}}^{t} H(t, s) \eta^{\Delta}(s) \frac{\omega(\sigma(s))}{\eta(\sigma(s))} \Delta s-\int_{t_{2}}^{t} H(t, s) \beta \frac{\eta(s)}{r^{1 / \beta}(s)} \frac{\omega^{\gamma}(\sigma(s))}{\eta^{\gamma}(\sigma(s))} \Delta s \\
\leq H\left(t, t_{2}\right) \omega\left(t_{2}\right)+\int_{t_{2}}^{t} \frac{h_{+}(t, s)}{\eta(\sigma(s))} H^{1 / \gamma}(t, s) \omega(\sigma(s)) \Delta s \\
-\int_{t_{2}}^{t} H(t, s) \beta \frac{\eta(s)}{r^{1 / \beta}(s)} \frac{\omega^{\gamma}(\sigma(s))}{\eta^{\gamma}(\sigma(s))} \Delta s .
\end{array}
$$

Applying Lemma 1.2 with

$X=\frac{[H(t, s) \beta \eta(s)]^{1 / \gamma}}{\left[r^{1 / \beta}(s) \eta^{\gamma}(\sigma(s))\right]^{1 / \gamma}} \omega(\sigma(s))$ and $Y=\left[\frac{\beta}{\beta+1} \frac{\left[r^{1 / \beta}(s) \eta^{\gamma}(\sigma(s))\right]^{1 / \gamma}}{[\beta \eta(s)]^{1 / \gamma}} \frac{h_{+}(t, s)}{\eta(\sigma(s))}\right]^{\beta}$

we obtain,

$$
\frac{h_{+}(t, s)}{\eta(\sigma(s))} H^{1 / \gamma}(t, s) \omega(\sigma(s))-H(t, s) \beta \frac{\eta(s)}{r^{1 / \beta}(s)} \frac{\omega^{\gamma}(\sigma(s))}{\eta^{\gamma}(\sigma(s))} \leq \frac{r(s)\left(h_{+}(t, s)\right)^{\beta+1}}{(\beta+1)^{\beta+1} \eta^{\beta}(s)} .
$$

Substituting the latter inequality into 2.20$)$, we conclude that

$$
\int_{t_{2}}^{t}\left[H(t, s) \Psi_{1}(s)-\frac{r(s)\left(h_{+}(t, s)\right)^{\beta+1}}{(\beta+1)^{\beta+1} \eta^{\beta}(s)}\right] \Delta s \leq H\left(t, t_{2}\right) \omega\left(t_{2}\right),
$$

which contradicts $(2.19)$. This proves the theorem.

From Theorem 2.2, we immediately have the following oscillation criterion.

Corollary 2.1. Suppose that all conditions of Theorem 2.2 are satisfied with 2.19) replaced by

$$
\limsup _{t \rightarrow \infty} \frac{1}{H\left(t, t_{*}\right)} \int_{t_{*}}^{t} H(t, s) \eta(s)\left(\sum_{i=1}^{n} q_{i}(s) \psi^{\beta}\left(h_{i}(s)\right) \frac{A^{\beta}\left(\tau_{2}^{-1}\left(h_{i}(s)\right), t_{1}\right)}{A^{\beta}\left(s, t_{1}\right)}\right) \Delta s=\infty
$$

and

$$
\limsup _{t \rightarrow \infty} \frac{1}{H\left(t, t_{*}\right)} \int_{t_{*}}^{t} \frac{r(s)\left(h_{+}(t, s)\right)^{\beta+1}}{\eta^{\beta}(s)} \Delta s<\infty,
$$

then equation (1.1) is oscillatory.

Theorem 2.3. Assume that conditions $(\mathfrak{i})-\left(\mathfrak{i i j}_{\mathfrak{a}}\right)$ hold and $h_{i}(t) \geq \tau_{2}(t)$ for $i=$ $1,2, \ldots, n$. If there exists a positive function $\eta \in C_{r d}^{1}\left(\left[t_{0}, \infty\right)_{\mathbb{T}}, \mathbb{R}\right)$ such that

$$
\limsup _{t \rightarrow \infty} \int_{T}^{t}\left(\eta(s)\left(\sum_{i=1}^{n} q_{i}(s) \psi^{\beta}\left(h_{i}(s)\right)\right)-\frac{r(s)\left(\eta^{\Delta}(s)\right)^{\beta+1}}{(\beta+1)^{\beta+1} \eta^{\beta}(s)}\right) \Delta s=\infty
$$


for $T>t_{1}$ with $t_{1} \in\left[t_{0}, \infty\right)_{\mathbb{T}}$ sufficiently large, then equation (1.1) is oscillatory.

Proof. Let $x(t)$ be a nonoscillatory solution of (1.1). Without loss of generality, we may assume that there exists $t_{1} \in\left[t_{0}, \infty\right)_{\mathbb{T}}$ such that $x(t)>0, x\left(\tau_{1}(t)\right)>0$, $x\left(\tau_{2}(t)\right)>0$ and $x\left(h_{i}(t)\right)>0$ for $t \geq t_{1}$ and $i=1,2, \ldots, n$. Proceeding as in the proof of Theorem 2.1, we again arrive at (2.11) for $t \geq t_{2}$. Using the fact that $\tau_{2}(t)$ is strictly increasing and noting that $h_{i}(t) \geq \tau_{2}(t)$, we have

$$
\tau_{2}^{-1}\left(h_{i}(t)\right) \geq t
$$

for $i=1,2, \ldots, n$. So, from the fact that $y$ is increasing, we obtain

$$
\frac{y\left(\tau_{2}^{-1}\left(h_{i}(t)\right)\right)}{y(t)} \geq 1, \quad i=1,2, \ldots, n .
$$

Using (2.23) in (2.11) yields

$$
\begin{aligned}
\omega^{\Delta}(t) \leq & \eta^{\Delta}(t) \frac{r(\sigma(t))\left(y^{\Delta}(\sigma(t))\right)^{\beta}}{y^{\beta}(\sigma(t))}-\eta(t) \frac{r(\sigma(t))\left(y^{\Delta}(\sigma(t))\right)^{\beta}\left(y^{\beta}(t)\right)^{\Delta}}{y^{\beta}(t) y^{\beta}(\sigma(t))} \\
& -\eta(t)\left(\sum_{i=1}^{n} q_{i}(t) \psi^{\beta}\left(h_{i}(t)\right)\right) \quad \text { for } t \geq t_{2} .
\end{aligned}
$$

The rest of proof is similar to that of Theorem 2.1 and so we omit the details.

Theorem 2.4. Assume that conditions $(\mathfrak{i})-\left(\mathfrak{i i i}_{\mathfrak{a}}\right)$ hold and $h_{i}(t) \geq \tau_{2}(t)$ for $i=$ $1,2, \ldots, n$. Suppose also that there exist functions $\eta \in C_{r d}^{1}\left(\left[t_{0}, \infty\right)_{\mathbb{T}}, \mathbb{R}^{+}\right)$and $H, h \in$ $C_{r d}(D, \mathbb{R})$ with $H$ belongs to the class $\mathcal{P}$ such that

$\limsup _{t \rightarrow \infty} \frac{1}{H\left(t, t_{*}\right)} \int_{t_{*}}^{t}\left[H(t, s) \eta(s)\left(\sum_{i=1}^{n} q_{i}(s) \psi^{\beta}\left(h_{i}(s)\right)\right)-\frac{r(s)\left(h_{+}(t, s)\right)^{\beta+1}}{(\beta+1)^{\beta+1} \eta^{\beta}(s)}\right] \Delta s=\infty$,

where $t_{*}>t_{1}$ for sufficiently large $t_{1} \in\left[t_{0}, \infty\right)_{\mathbb{T}}$. Then every solution of equation (1.1) is oscillatory.

Proof. The proof follows from 2.23, 2.24 and Theorem 2.2 and so we omit the details.

\section{Oscillation Results When ( $\mathfrak{i i}_{\mathfrak{b}}$ ) HOLDS}

In this section, we establish some new criteria for the oscillation of (1.1) in the cases where $h_{i}(t) \leq \tau_{1}(t)$ and $h_{i}(t) \geq \tau_{1}(t)$ for $i=1,2, \ldots, n$, respectively. For notational purposes, we let

$$
\begin{array}{r}
\phi(t):=\frac{1}{p_{1}\left(\tau_{1}^{-1}(t)\right)}\left[1-\frac{1}{p_{1}\left(\tau_{1}^{-1}\left(\tau_{1}^{-1}(t)\right)\right)} \frac{A\left(\tau_{1}^{-1}\left(\tau_{1}^{-1}(t)\right), t_{1}\right)}{A\left(\tau_{1}^{-1}(t), t_{1}\right)}\right. \\
\left.-\frac{p_{2}\left(\tau_{1}^{-1}(t)\right)}{p_{1}\left(\tau_{1}^{-1}\left(\tau_{2}\left(\tau_{1}^{-1}(t)\right)\right)\right)} \frac{A\left(\tau_{1}^{-1}\left(\tau_{2}\left(\tau_{1}^{-1}(t)\right)\right), t_{1}\right)}{A\left(\tau_{1}^{-1}(t), t_{1}\right)}\right]
\end{array}
$$


where $\tau_{1}^{-1}$ denotes the inverse function of $\tau_{1}$, and throughout this section we assume that $\phi(t)>0$ for all sufficiently large $t$.

Theorem 3.1. Suppose that conditions $(\mathfrak{i})$, (ii), ( $\left.\mathfrak{i i}_{\mathfrak{b}}\right)$ hold and $h_{i}(t) \leq \tau_{1}(t)$ for $i=1,2, \ldots, n$. If there exists a positive function $\eta \in C_{r d}^{1}\left(\left[t_{0}, \infty\right)_{\mathbb{T}}, \mathbb{R}\right)$ such that

$$
\limsup _{t \rightarrow \infty} \int_{T}^{t}\left(\Psi_{2}(s)-\frac{r(s)\left(\eta^{\Delta}(s)\right)^{\beta+1}}{(\beta+1)^{\beta+1} \eta^{\beta}(s)}\right) \Delta s=\infty
$$

for $T>t_{1}$ where $t_{1} \in\left[t_{0}, \infty\right)_{\mathbb{T}}$ sufficiently large and

$$
\Psi_{2}(t)=\eta(t)\left(\sum_{i=1}^{n} q_{i}(t) \phi^{\beta}\left(h_{i}(t)\right) \frac{A^{\beta}\left(\tau_{1}^{-1}\left(h_{i}(t)\right), t_{1}\right)}{A^{\beta}\left(t, t_{1}\right)}\right),
$$

then equation (1.1) is oscillatory.

Proof. Let $x(t)$ be a nonoscillatory solution of $(1.1)$. Without loss of generality, we may assume that there exists $t_{1} \in\left[t_{0}, \infty\right)_{\mathbb{T}}$ such that $x(t)>0, x\left(\tau_{1}(t)\right)>0$, $x\left(\tau_{2}(t)\right)>0$ and $x\left(h_{i}(t)\right)>0$ for $t \geq t_{1}$ and $i=1,2, \ldots, n$. Following a similar procedure to the proof of Theorem 2.1, we see that 2.2 , 2.3 hold again, and from the definition of $y(t)$, we obtain

$x(t) \geq \frac{y\left(\tau_{1}^{-1}(t)\right)}{p_{1}\left(\tau_{1}^{-1}(t)\right)}-\frac{y\left(\tau_{1}^{-1}\left(\tau_{1}^{-1}(t)\right)\right)}{p_{1}\left(\tau_{1}^{-1}(t)\right) p_{1}\left(\tau_{1}^{-1}\left(\tau_{1}^{-1}(t)\right)\right)}-\frac{p_{2}\left(\tau_{1}^{-1}(t)\right) y\left(\tau_{1}^{-1}\left(\tau_{2}\left(\tau_{1}^{-1}(t)\right)\right)\right)}{p_{1}\left(\tau_{1}^{-1}(t)\right) p_{1}\left(\tau_{1}^{-1}\left(\tau_{2}\left(\tau_{1}^{-1}(t)\right)\right)\right)}$.

Using the fact that $\tau_{1}$ and $\tau_{2}$ are strictly increasing, and noting that $\tau_{1}(t)<t<$ $\tau_{2}(t)$, we get

$$
\tau_{1}^{-1}\left(\tau_{1}^{-1}(t)\right)>\tau_{1}^{-1}(t)
$$

and

$$
\tau_{1}^{-1}\left(\tau_{2}\left(\tau_{1}^{-1}(t)\right)\right)>\tau_{1}^{-1}(t)
$$

Taking $y(t) / A\left(t, t_{1}\right)$ is nonincreasing on $\left[t_{1}, \infty\right)_{\mathbb{T}}$ into account that, we deduce from (3.3) and 3.4 that

$$
\frac{A\left(\tau_{1}^{-1}\left(\tau_{1}^{-1}(t)\right), t_{1}\right) y\left(\tau_{1}^{-1}(t)\right)}{A\left(\tau_{1}^{-1}(t), t_{1}\right)} \geq y\left(\tau_{1}^{-1}\left(\tau_{1}^{-1}(t)\right)\right) \quad \text { for } t \geq t_{1},
$$

and

$$
\frac{A\left(\tau_{1}^{-1}\left(\tau_{2}\left(\tau_{1}^{-1}(t)\right)\right), t_{1}\right) y\left(\tau_{1}^{-1}(t)\right)}{A\left(\tau_{1}^{-1}(t), t_{1}\right)} \geq y\left(\tau_{1}^{-1}\left(\tau_{2}\left(\tau_{1}^{-1}(t)\right)\right)\right) \quad \text { for } t \geq t_{1},
$$

respectively. Using 3.5 and 3.6 in 3.4 yields

$$
x(t) \geq \frac{1}{p_{1}\left(\tau_{1}^{-1}(t)\right)}\left[1-\frac{1}{p_{1}\left(\tau_{1}^{-1}\left(\tau_{1}^{-1}(t)\right)\right)} \frac{A\left(\tau_{1}^{-1}\left(\tau_{1}^{-1}(t)\right), t_{1}\right)}{A\left(\tau_{1}^{-1}(t), t_{1}\right)}\right.
$$




$$
\left.-\frac{p_{2}\left(\tau_{1}^{-1}(t)\right)}{p_{1}\left(\tau_{1}^{-1}\left(\tau_{2}\left(\tau_{1}^{-1}(t)\right)\right)\right)} \frac{A\left(\tau_{1}^{-1}\left(\tau_{2}\left(\tau_{1}^{-1}(t)\right)\right), t_{1}\right)}{A\left(\tau_{1}^{-1}(t), t_{1}\right)}\right] y\left(\tau_{1}^{-1}(t)\right)
$$

for $t \geq t_{1}$. Since $\lim _{t \rightarrow \infty} h_{i}(t)=\infty$, we can choose $t_{2} \geq t_{1}$ such that all $h_{i}(t) \geq t_{1}$ for $t \geq t_{2}$, where $i=1,2, \ldots, n$. Thus, from (3.7) we obtain

$$
x\left(h_{i}(t)\right) \geq \phi\left(h_{i}(t)\right) y\left(\tau_{1}^{-1}\left(h_{i}(t)\right)\right), \quad i=1,2, \ldots, n
$$

for $t \geq t_{2}$. Using (3.8) in (1.1) gives

$$
\left(r(t)\left(y^{\Delta}(t)\right)^{\beta}\right)^{\Delta}+\sum_{i=1}^{n} q_{i}(t) \phi^{\beta}\left(h_{i}(t)\right) y^{\beta}\left(\tau_{1}^{-1}\left(h_{i}(t)\right)\right) \leq 0 .
$$

Define again Riccati substitution $\omega$ by 2.10 . Then, it follows from 2.10 and 3.9 ) that

$$
\begin{aligned}
\omega^{\Delta}(t) \leq & \eta^{\Delta}(t) \frac{r(\sigma(t))\left(y^{\Delta}(\sigma(t))\right)^{\beta}}{y^{\beta}(\sigma(t))}-\eta(t) \frac{r(\sigma(t))\left(y^{\Delta}(\sigma(t))\right)^{\beta}\left(y^{\beta}(t)\right)^{\Delta}}{y^{\beta}(t) y^{\beta}(\sigma(t))} \\
& -\eta(t)\left(\sum_{i=1}^{n} q_{i}(t) \phi^{\beta}\left(h_{i}(t)\right) \frac{y^{\beta}\left(\tau_{1}^{-1}\left(h_{i}(t)\right)\right)}{y^{\beta}(t)}\right) \quad \text { for } t \geq t_{2} .
\end{aligned}
$$

Using the fact $y(t) / A\left(t, t_{1}\right)$ is nonincreasing, and noting that $h_{i}(t) \leq \tau_{1}(t)$ implies $\tau_{1}^{-1}\left(h_{i}(t)\right) \leq t$, we obtain

$$
\frac{y\left(\tau_{1}^{-1}\left(h_{i}(t)\right)\right)}{y(t)} \geq \frac{A\left(\tau_{1}^{-1}\left(h_{i}(t)\right), t_{1}\right)}{A\left(t, t_{1}\right)} .
$$

for $i=1,2, \ldots, n$. Substituting (3.11) into 3.10) gives

$$
\begin{aligned}
\omega^{\Delta}(t) \leq & \eta^{\Delta}(t) \frac{r(\sigma(t))\left(y^{\Delta}(\sigma(t))\right)^{\beta}}{y^{\beta}(\sigma(t))}-\eta(t) \frac{r(\sigma(t))\left(y^{\Delta}(\sigma(t))\right)^{\beta}\left(y^{\beta}(t)\right)^{\Delta}}{y^{\beta}(t) y^{\beta}(\sigma(t))} \\
& -\eta(t)\left(\sum_{i=1}^{n} q_{i}(t) \phi^{\beta}\left(h_{i}(t)\right) \frac{A^{\beta}\left(\tau_{1}^{-1}\left(h_{i}(t)\right), t_{1}\right)}{A^{\beta}\left(t, t_{1}\right)}\right) \quad \text { for } t \geq t_{2} .
\end{aligned}
$$

The remainder of the proof is similar to that of Theorem 2.1, and so the details are omitted.

Theorem 3.2. Suppose that conditions $(\mathfrak{i})$, (ii), $\left(\mathfrak{i i}_{\mathfrak{b}}\right)$ hold and $h_{i}(t) \leq \tau_{1}(t)$ for $i=1,2, \ldots, n$. Suppose also that there exist functions $\eta \in C_{r d}^{1}\left(\left[t_{0}, \infty\right)_{\mathbb{T}}, \mathbb{R}^{+}\right)$and $H, h \in C_{r d}(D, \mathbb{R})$ with $H$ belongs to the class $\mathcal{P}$ such that

$$
\limsup _{t \rightarrow \infty} \frac{1}{H\left(t, t_{*}\right)} \int_{t_{*}}^{t}\left[H(t, s) \Psi_{2}(s)-\frac{r(s)\left(h_{+}(t, s)\right)^{\beta+1}}{(\beta+1)^{\beta+1} \eta^{\beta}(s)}\right] \Delta s=\infty,
$$

where $\Psi_{2}(t)$ is as in Theorem 3.1 and $t_{*}>t_{1}$ for sufficiently large $t_{1} \in\left[t_{0}, \infty\right)_{\mathbb{T}}$. Then every solution of equation (1.1) is oscillatory. 
Proof. The proof follows from 3.11, 3.12 and Theorem 2.2, and so we omit the details.

From Theorem 3.3 , we immediately have the following oscillation criterion.

Corollary 3.1. Suppose that all conditions of Theorem 3.2 are satisfied with (3.13) replaced by

$$
\limsup _{t \rightarrow \infty} \frac{1}{H\left(t, t_{*}\right)} \int_{t_{*}}^{t} H(t, s) \eta(s)\left(\sum_{i=1}^{n} q_{i}(s) \phi^{\beta}\left(h_{i}(s)\right) \frac{A^{\beta}\left(\tau_{1}^{-1}\left(h_{i}(s)\right), t_{1}\right)}{A^{\beta}\left(s, t_{1}\right)}\right) \Delta s=\infty
$$

and

$$
\limsup _{t \rightarrow \infty} \frac{1}{H\left(t, t_{*}\right)} \int_{t_{*}}^{t} \frac{r(s)\left(h_{+}(t, s)\right)^{\beta+1}}{\eta^{\beta}(s)} \Delta s<\infty,
$$

then equation 1.1) is oscillatory.

Theorem 3.3. Assume that conditions $(\mathfrak{i})$, (ii), $\left(\mathfrak{i i i}_{\mathfrak{b}}\right)$ hold and $h_{i}(t) \geq \tau_{1}(t)$ for $i=1,2, \ldots, n$. If there exists a positive function $\eta \in C_{r d}^{1}\left(\left[t_{0}, \infty\right)_{\mathbb{T}}, \mathbb{R}\right)$ such that

$$
\limsup _{t \rightarrow \infty} \int_{T}^{t}\left(\eta(s)\left(\sum_{i=1}^{n} q_{i}(s) \phi^{\beta}\left(h_{i}(s)\right)\right)-\frac{r(s)\left(\eta^{\Delta}(s)\right)^{\beta+1}}{(\beta+1)^{\beta+1} \eta^{\beta}(s)}\right) \Delta s=\infty,
$$

for $T>t_{1}$ with $t_{1} \in\left[t_{0}, \infty\right)_{\mathbb{T}}$ sufficiently large, then equation 1.1$]$ is oscillatory.

Proof. Let $x(t)$ be a nonoscillatory solution of (1.1). Without loss of generality, we may assume that there exists $t_{1} \in\left[t_{0}, \infty\right)_{\mathbb{T}}$ such that $x(t)>0, x\left(\tau_{1}(t)\right)>0$, $x\left(\tau_{2}(t)\right)>0$ and $x\left(h_{i}(t)\right)>0$ for $t \geq t_{1}$ and $i=1,2, \ldots, n$. Proceeding as in the proof of Theorem 3.1. we again arrive at 3.10 for $t \geq t_{2}$. Using the fact that $\tau_{1}(t)$ is strictly increasing and noting that $h_{i}(t) \geq \tau_{1}(t)$, we have

$$
\tau_{1}^{-1}\left(h_{i}(t)\right) \geq t
$$

for $i=1,2, \ldots, n$. So, from the fact that $y$ is increasing, we obtain

$$
\frac{y\left(\tau_{1}^{-1}\left(h_{i}(t)\right)\right)}{y(t)} \geq 1, \quad i=1,2, \ldots, n .
$$

Using (3.15) in 3.10 yields

$$
\begin{aligned}
\omega^{\Delta}(t) \leq & \eta^{\Delta}(t) \frac{r(\sigma(t))\left(y^{\Delta}(\sigma(t))\right)^{\beta}}{y^{\beta}(\sigma(t))}-\eta(t) \frac{r(\sigma(t))\left(y^{\Delta}(\sigma(t))\right)^{\beta}\left(y^{\beta}(t)\right)^{\Delta}}{y^{\beta}(t) y^{\beta}(\sigma(t))} \\
& -\eta(t)\left(\sum_{i=1}^{n} q_{i}(t) \phi^{\beta}\left(h_{i}(t)\right)\right) \quad \text { for } t \geq t_{2} .
\end{aligned}
$$

The rest of proof is similar to the proof of Theorem 2.1. and so the details are omitted. 
Theorem 3.4. Suppose that conditions $(\mathfrak{i})$, (ii), $\left(\mathfrak{i i}_{\mathfrak{b}}\right)$ hold and $h_{i}(t) \geq \tau_{1}(t)$ for $i=1,2, \ldots, n$. Suppose also that there exist functions $\eta \in C_{r d}^{1}\left(\left[t_{0}, \infty\right)_{\mathbb{T}}, \mathbb{R}^{+}\right)$and $H, h \in C_{r d}(D, \mathbb{R})$ with $H$ belongs to the class $\mathcal{P}$ such that

$\limsup _{t \rightarrow \infty} \frac{1}{H\left(t, t_{*}\right)} \int_{t_{*}}^{t}\left[H(t, s) \eta(s)\left(\sum_{i=1}^{n} q_{i}(s) \phi^{\beta}\left(h_{i}(s)\right)\right)-\frac{r(s)\left(h_{+}(t, s)\right)^{\beta+1}}{(\beta+1)^{\beta+1} \eta^{\beta}(s)}\right] \Delta s=\infty$,

where $t_{*}>t_{1}$ for sufficiently large $t_{1} \in\left[t_{0}, \infty\right)_{\mathbb{T}}$. Then every solution of equation (1.1) is oscillatory.

Proof. The proof follows from (3.15), (3.16) and Theorem 2.2 and so we omit the details.

\section{Examples and Remarks}

Example 4.1. Consider the neutral differential equation

$$
\left[\left(\left(x(t)+x\left(\frac{t}{2}\right)+t x(2 t)\right)^{\prime}\right)^{3}\right]^{\prime 4} x^{3}(t-1)+t^{5} x^{3}(2 t-1)=0
$$

for $t \geq 13$. Here we have $\mathbb{T}=\mathbb{R}, \beta=3, n=2, r(t)=p_{1}(t)=1, p_{2}(t)=t$, $q_{1}(t)=t^{4}, q_{2}(t)=t^{5}, \tau_{1}(t)=t / 2, \tau_{2}(t)=2 t, h_{1}(t)=t-1$ and $h(t)=2 t-1$. It is clear that conditions $(\mathfrak{i})-\left(\mathfrak{i i j}_{\mathfrak{a}}\right)$ hold, $\tau_{2}(t)>h_{2}(t)>t>h_{1}(t)$ and

$$
\psi(t)=\frac{1}{t / 2}\left[1-\frac{1}{t / 4}-\frac{1}{t / 8}\right]=\frac{2 t-24}{t^{2}}>0, \quad \text { for } t \geq 13 .
$$

On the other hand, we see that

$$
A\left(t, t_{1}\right)=\int_{t_{1}}^{t} \frac{1}{r^{1 / \beta}(s)} d s=\int_{13}^{t} d s=t-13 .
$$

With $\eta(t)=t$, condition 2.1) is satisfied due to

$$
\limsup _{t \rightarrow \infty} \int_{T}^{t}\left[s^{5}\left(\frac{2 s-26}{(s-1)^{2}}\right)^{3}\left(\frac{s-27}{2 s-26}\right)^{3}+s^{6}\left(\frac{4 s-26}{(2 s-1)^{2}}\right)^{3}\left(\frac{s-27 / 2}{s-13}\right)^{3}\right] d s=\infty
$$

and

$$
\limsup _{t \rightarrow \infty} \int_{T}^{t} \frac{1}{4^{4} s^{3}} d s<\infty
$$

where $T>13$. Hence, by Theorem 2.1, every solution of 4.1 is oscillatory.

Example 4.2. Let $\mathbb{T}=\mathbb{Z}$ and consider the neutral difference equation

$$
\Delta\left[\frac{1}{t^{1 / 3}}(\Delta(x(t)+3 x(t-3)+7 x(t+1)))^{1 / 3}\right]+\sum_{i=1}^{n} t^{i} x^{1 / 3}(t+i+1)=0,
$$


for $t \geq 2$. Here we have $\beta=1 / 3, r(t)=1 / t^{1 / 3}, p_{1}(t)=3, p_{2}(t)=7, \tau_{1}(t)=t-3$, $\tau_{2}(t)=t+1, q_{i}(t)=t^{i}$ and $h_{i}(t)=t+i+1$ for $i=1,2, \ldots, n$. It is clear that conditions (i)-(iii $\left.\mathfrak{i}_{\mathfrak{a}}\right)$ hold, $t<\tau_{2}(t)<h_{i}(t)$ for $i=1,2, \ldots, n$ and

$$
\psi(t)=\frac{1}{7}\left[1-\frac{1}{7}-\frac{3}{7}\right]=\frac{3}{49}>0 .
$$

It is easy to see that condition 2.22 holds with $\eta(t)=c>0$. So, by Theorem 2.3 . all solutions of equation 4.3 are oscillatory.

Example 4.3. Consider the second order neutral dynamic equation

$$
\left(\left(\left(x(t)+5 t x\left(\frac{t}{2}\right)+t x(4 t)\right)^{\Delta}\right)^{3}\right)^{\Delta}+t^{2} x^{3}\left(\frac{t}{4}\right)+t^{3} x^{3}\left(\frac{t}{8}\right)=0,
$$

for $t \geq 2$ and $\mathbb{T}:=\overline{2^{\mathbb{Z}}}=\left\{2^{k}: k \in \mathbb{Z}\right\} \cup\{0\}$. Here we have $\beta=3, n=2, r(t)=1$, $p_{1}(t)=5 t, p_{2}(t)=t, \tau_{1}(t)=t / 2, \tau_{2}(t)=4 t, q_{1}(t)=t^{2}, q_{2}(t)=t^{3}, h_{1}(t)=t / 4$ and $h_{2}(t)=t / 8$. It is clear that conditions $(\mathfrak{i}),(\mathfrak{i i}),\left(\mathfrak{i i i}_{\mathfrak{b}}\right)$ hold and $\tau_{1}(t) \geq h_{i}(t)$ for $i=1,2$. Also, it obvious that $A\left(t, t_{1}\right)=t-2$ for any time scale $\mathbb{T}$, and

$$
\phi(t)=\frac{1}{10 t}\left[1-\frac{1}{20 t} \frac{4 t-2}{2 t-2}-\frac{2 t}{80 t} \frac{16 t-2}{2 t-2}\right]=\frac{32 t^{2}-43 t+2}{400 t^{3}-400 t^{2}}>0
$$

for $t \geq 2$. Then, with $\eta(t)=t^{2}$, we see that condition (3.1) holds for $T>2$. Hence, by Theorem 3.1. every solution of second order mixed neutral q-difference equation 4.5 is oscillatory.

Example 4.4. Consider the neutral differential equation

$$
(x(t)+t x(t-2 \pi)+x(t+\pi))^{\prime \prime}+2 x\left(t-\frac{\pi}{2}\right)+t x(t+2 \pi)=0, \quad t \geq 5 .
$$

Here we have $\mathbb{T}=\mathbb{R}, \beta=1, n=2, r(t)=p_{2}(t)=1, p_{1}(t)=t, \tau_{1}(t)=t-2 \pi$, $\tau_{2}(t)=t+\pi, q_{1}(t)=2, q_{2}(t)=t, h_{1}(t)=t-\frac{\pi}{2}$ and $h_{2}(t)=t+2 \pi$. It is clear that conditions $(\mathfrak{i}),(\mathfrak{i i}),\left(\mathfrak{i i i}_{\mathfrak{b}}\right)$ hold, and $\tau_{1}(t)<h_{1}(t)<h_{2}(t)$. On the other hand, we see that $A\left(t, t_{1}\right)=t-5$, and $\phi(t)>0$. With $\eta(t)=1$, it is easy to see that condition (3.14) holds. Hence, by Theorem 3.3 every solution of equation (4.7) is oscillatory. In fact, $x(t)=\sin t$ is such a solution.

Remark 4.1. Note that oscillation results presented in 6, 10, 12, 16, 19, 23, 28, 30, 36, 37. fail to apply to the equations 4.1), 4.3), 4.5) and (4.7).

\section{REFERENCES}

[1] Agarwal, R. P., Grace, S. R. and O'Regan, D., Oscillation criteria for certain nth order differential equations with deviating arguments, J. Math. Appl. Anal., 262 (2001), 601-622.

[2] Agarwal, R. P., Grace, S. R. and O'Regan, D., The oscillation of certain higher-order functional dfferential equations, Math. Comput. Model., 37 (2003), 705-728.

[3] Agarwal, R. P., O'Regan, D. and Saker, S. H., Philos-type oscillation criteria for second order half linear dynamic equations, Rocky Mountain J. Math., 37 (2007), 1085-1104. 
[4] Agarwal, R. P., Bohner, M., Li, T. and Zhang, C., A Philos-type theorem for third-order nonlinear retarded dynamic equations, Appl. Math. Comput., 249 (2014), 527-531.

[5] Agarwal, R. P., Bohner, M., Li, T. and Zhang, C., Oscillation criteria for second-order dynamic equations on time scales, Appl. Math. Lett., 31 (2014), 34-40.

[6] Agwa, H. A., Khodier, A. M. M. and Arafa, H. M., Oscillation of second-order nonlinear neutral dynamic equations with mixed arguments on time scales, Journal of Basic and Applied Research International, 17 (2016), 49-66.

[7] Baculíková, B. and Džurina, J., Oscillation theorems for second-order nonlinear neutral differential equations, Comput. Math. Appl., 62(12) (2011), 4472-4478.

[8] Bohner, M. and Peterson, A., Dynamic Equations on Time Scales. An Introduction with Applications, Birkhäuser, Boston, 2001.

[9] Bohner, M. and Peterson, A., Advances in Dynamic Equations on Time Scales, Birkhäuser, Boston, 2003.

[10] Candan, T. and Dahiya, R. S., On the oscillation of certain mixed neutral equations, Appl. Math. Lett., 21 (2008), 222-226.

[11] Chatzarakis, G. E. and Miliaras, G. N., Covergence of the solutions for a neutral difference equation with negative coefficients, Tatra Mt. Math. Publ., 54 (2013), 31-43.

[12] Grace, S. R., On the oscillations of mixed neutral equations, J. Math. Anal. Appl., 194 (1995), 377-388.

[13] Grace, S. R., Džurina, J., Jadlovská, I. and Li, T., An improved approach for studying oscillation of second-order neutral delay differential equations, J. Inequal. Appl., 2018:193 (2018), 13 pages.

[14] Graef, J. R., Tunç, E. and Grace, S. R., Oscillatory and asymptotic behavior of a third-order nonlinear neutral differential equation, Opuscula Math., 37(6) (2017), 839-852.

[15] Hale, J., Theory of Functional Differential Equations, Springer-Verlag, New York, 1977.

[16] Han, Z., Li, T., Zhang, C. and Sun, Y., Oscillation criteria for certain second-order nonlinear neutral differential equations of mixed type, Abstr. Appl. Anal., 2011 (2011), Article ID: $387483,1-9$

[17] Hardy, G. H., Littlewood, J. E. and Polya, G., Inequalities, Reprint of the 1952 edition, Cambridge University Press, Cambridge, 1988.

[18] Hilger, S., Analysis on measure chains A unified approach to continuous and discrete calculus, Results Math., 18 (1990), 18-56.

[19] Ji, T., Tang, S. and Thandapani, E., Oscillation of second-order neutral dynamic equations with mixed arguments, Appl. Math. Inf. Sci., 8 (2014), 2225-2228.

[20] Karpuz, B., Manojlović, J. V., Öcalan, Ö. and Shoukaku, Y., Oscillation criteria for a class of second-order neutral delay differential equations, Appl. Math. Comput., 210 (2009), 303-312.

[21] Karpuz, B., Öcalan, Ö. and Yıldız, M. K., Oscillation of a class of difference equations of second order, Math. Comput. Model., 49 (2009), 912-917.

[22] Kolmanovskii, V. and Myshkis, A., Introduction to the Theory and Applications of Functional Differential Equations, Kluwer Academic Publishers, Dordrecht, 1999.

[23] Li, T., Comparison theorems for second-order neutral differential equations of mixed type, Electron. J. Differ. Eq., 2010 (2010), No. 167, 1-7.

[24] Li, T., Agarwal, R. P. and Bohner, M., Some oscillation results for second-order neutral dynamic equations, Hacet. J. Math. Stat., 41 (2012), 715-721.

[25] MacDonald, N., Biological Delay Systems: Linear Stability Theory, Cambridge University Press, Cambridge, 1989.

[26] Özdemir, O. and Tunç, E., Asymptotic behavior and oscillation of solutions of third order neutral dynamic equations with distributed deviating arguments, Bull. Math. Anal. Appl., 10(2) (2018), 31-52.

[27] Philos, Ch. G., Oscillation theorems for linear differential equations of second order, Arch. Math., 53 (1989), 482-492. 
[28] Qi, Y. and Yu, J., Oscillation of second order nonlinear mixed neutral differential equations with distributed deviating arguments, Bull. Malays. Math. Sci. Soc., 38 (2015), 543-560.

[29] Saker, S. H., Oscillation of second-order nonlinear neutral delay dynamic equations on time scales, J. Comput. Appl. Math., 187 (2006), 123-141.

[30] Thandapani, E., Padmavathi, S. and Pinelas, S., Oscillation criteria for even-order nonlinear neutral differential equations of mixed type, Bull. Math. Anal. Appl., 6(1) (2014), 9-22.

[31] Tunç, E. and Graef, J. R., Oscillation results for second order neutral dynamic equatitons with distributed deviating arguments, Dynam. Syst. Appl., 23(2-3) (2014), 289-303.

[32] Tunç, E. and Grace, S. R., On oscillatory and asymptotic behavior of a second order nonlinear damped neutral differential equation, International Journal of Differential Equations, 2016 (2016), Article ID: 3746368, 8 pages.

[33] Tunç, E. and Özdemir, O., On the asymptotic and oscillatory behavior of solutions of thirdorder neutral dynamic equations on time scales, Adv. Difference Equ., 2017:127 (2017), 13 pages.

[34] Tunç, E. and Liu, H., Oscillatory behavior for second-order damped differential equation with nonlinearities including Riemann-Stieltjes integrals, Electron. J. Differ. Equ., 2018(54) (2018), 1-12.

[35] Tunç, E. and Özdemir, O., On the oscillation of second-order half-linear functional differential equations with mixed neutral term, J. Taibah Univ. Sci., 13(1) (2019), 481-489.

[36] Yan, J., Oscillations of second order neutral functional differential equations, Appl. Math. Comput., 83 (1997), 27-41.

[37] Zhang, C., Baculíková, B., Džurina, J. and Li, T., Oscillation results for second-order mixed neutral differential equations with distributed deviating arguments, Math. Slovaca, 66(3) (2016), 615-626.

Current address: Orhan Özdemir: Department of Mathematics, Faculty of Arts and Sciences, Gaziosmanpasa University, 60240, Tokat, Turkey

E-mail address: orhanozdemir37@yahoo.com

ORCID Address: http://orcid.org/0000-0003-1294-5346 